\title{
TENDON GRAFTING
}

\section{Illustrated by a New Operation for Intrinsic Paralysis of the Fingers}

\author{
Paul W. Brand, Vellore, India \\ Professor of Orthopaedic Surgery, Christian Medical College and Hospital, Vellore
}

\begin{abstract}
A free tendon graft always becomes adherent. A tendon in living continuity with its muscle may develop a new gliding path completely free of adhesions after being transferred. A free graft, because it has to acquire a new blood supply, has to become connected to the surrounding structures. One should, therefore, choose to transfer rather than graft a tendon, when other considerations do not limit one's choice, such as there being no unparalysed muscle with a tendon long enough to reach the required insertion, or that the transfer of a suitable tendon would leave a weakness in the donor function. Again, in operations to balance the muscle power of the fingers, the muscles that move the wrist are a valuable source of power, but they have to be lengthened by a tendon graft.

The adhesions that occur after a tendon graft limit the movement of the tendon in proportion to their density, and in inverse proportion to the mobility of the normal structures to which they are attached. Although their differences are mainly of degree they may be considered in two groups: scar adhesions and blood vessel adhesions. The former are rigid and crippling, while the latter vary in their effect, and will stretch after operation to allow fair mobility.
\end{abstract}

Scar adhesions-Every wound heals with a scar. The scar is common to the whole wound and connects each and every structure in the wound. The density of the scar varies with the skill of the operator, with the amount of local trauma, and with the presence or absence of haematomata and infection. This scar, whether it is thick or thin, causes a direct adhesion between every immobile structure that has been divided in the wound and the structures which are planned to move.

The worst adhesion of all is that which grows from a tendon anastomosis; it is a product not of the graft but of the living motor tendon. Bunnell (1932) has vividly described its nature, and calls it a pseudopodium which grows out from an " unsatisfied" end of a tendon. Bunnell emphasises the importance of ensuring that every fibre of tendon that has been cut should be " satisfied" by immediate attachment to another cut surface, whether tendon or bone. When operating after a previous reconstruction has failed, we have often had the humbling experience of confirming the accuracy of Bunnell's description. Even after careful end-to-end suture of tendons, it is sometimes found that a stray fibre has escaped the suture line to form an anchor. An adhesion from the unsatisfied end of a tendon is so crippling because of the purposeful way in which it grows on and on, like the tendril of a vine that is seeking support, until it attaches itself to a rigid structure. Once anchored, the growing fibre thickens and contracts and draws the tendon anastomosis towards this new insertion, to rob the tendon graft of all mobility.

It is very difficult to avoid an unsatisfied end of a thick motor tendon when a thin graft is being attached to it. Under such circumstances Bunnell has suggested " bird-beaking" the thicker tendon to reduce its diameter, and Pulvertaft (1956) has used adjacent structures such as the lumbrical muscle belly in the palm to cover the anastomosis. A new method of tendon anastomosis which eliminates the danger of the unsatisfied end is described below.

Blood vessel adhesions-Suppose that no rigid structure has been divided and that a free graft lies in a snug tunnel that has been bloodlessly created by the gentle pressure of a blunt-nosed tunneller in a connective-tissue plane. Even under such ideal circumstances the graft cannot live without the blood vessels that must grow into its surface from the surrounding tissue. 
These vessels, although easier to stretch than the scar tissue, constitute a drag on the tendon, and without disciplined exercises after operation they may greatly limit movement.

Final range of movement-In the absence of rigid adhesions the final range of movement obtained by a tendon graft is determined by two factors: the movement allowed by the length and stretch of blood vessel adhesions, and the movement of the adjacent tissues to which these adhesions are attached. Though it is difficult to separate these two factors in any one case, we have recently demonstrated in a series of experiments in monkeys that in many successful grafts the second factor has accounted for the greater part of the movement. The nature of the tissues lining its path may decide success or failure to a greater extent for a tendon graft than for a tendon transfer.

In addition to the basic rules for tendon transfers (Mayer 1916) there are, therefore, rules which are of special importance for tendon grafting. 1) Scarring must be kept to the least possible by atraumatic technique and careful haemostasis. 2) The extent of the scar should be kept small by the use of small incisions with tunnels made by a blunt instrument between them. Fortunately in tendon grafting it is usually possible to do the tendon anastomosis outside the limb without raising a flap. The motor tendon is brought out through a proximal transverse incision. The graft, after attachment to the motor tendon, is passed through a tunnel to its insertion, pulling the anastomosis behind it into an undamaged gliding plane away from the incision. This is not possible in direct tendon repair, where the anastomosis has to lie in the wound of access. 3) Mobile structures must surround the tendon graft. Conversely, no rigid structure should be divided or even scratched in the same wound as a tendon

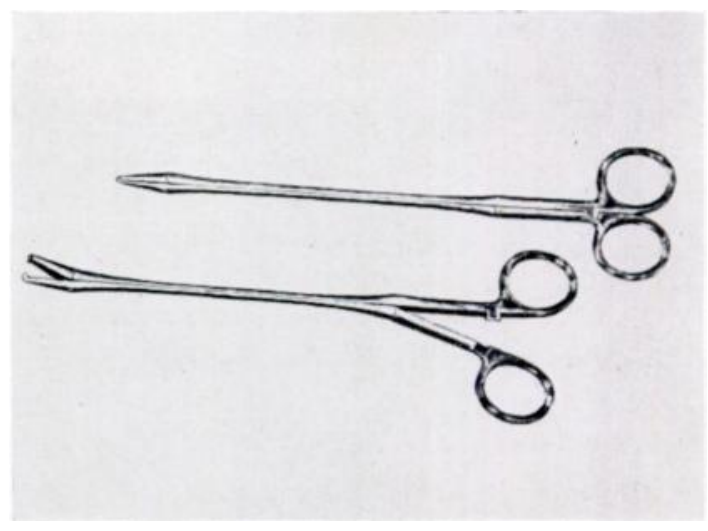

FiG. 1

Tendon tunneller (J. G. Andersen).

graft. When a tendon has to pass near bone, ligament or retinaculum, or between fibrous septa linking the skin to a deeper, immobile plane, the tunnel should be made gently by the blunt-nosed tunnelling forceps (Fig. 1). The surgeon must practise the patience of an earthworm feeling its way between roots and stones, and he must not force a way through rigid structures or the tunnel will not be lined with yielding material. The closeness of a rigid structure does not matter, so long as it is not wounded. Most rigid structures are surrounded by an almost invisible layer of slippery tissue, which, if it has not been scarred, is remarkably elastic. It is the unavoidable proximity of rigid structures in the flexor sheath of the finger that makes this area a difficult problem, with its own special rules. Here we follow Pulvertaft's (1956) method. 4) Tunnels must not be made larger than necessary. It is false reasoning to expect extra space to allow greater freedom for the graft, for this extra space becomes filled with scar, which will allow less mobility than almost any intact tissue.

\section{TECHNIQUE OF ANASTOMOSIS OF TENDONS OF UNEQUAL THICKNESS}

Crippling adhesions grow from the cut end of a tendon. A longitudinal slit heals with almost no adhesions. The following technique is based on the principle that all cut ends may be wrapped up in sheets made from opened-out tendon (Fig. 2).

Most tendons are easily opened into flat sheets, without damage to their gliding surfaces, and many tendons possess the structure of a rolled-up sheet. The free edge of the sheet is marked by a visible cleft or hilum on the deep surface of the tendon into which fatty, elastic strands carry nutrient blood vessels. If a knife blade is slipped along such a cleft the tendon 
can be opened out and unrolled. Even if a natural plane of cleavage is not obvious, a thick tendon may be slit open and a tendon graft may be laid inside it and stitched in position, after which the stouter tendon is rolled up, and the edges are held together by a running filament of 45 gauge stainless steel wire.

If more than one graft is to be attached to one motor tendon the end of each may enter the opened-out motor tendon through a separate longitudinal slit so that not more than one

I

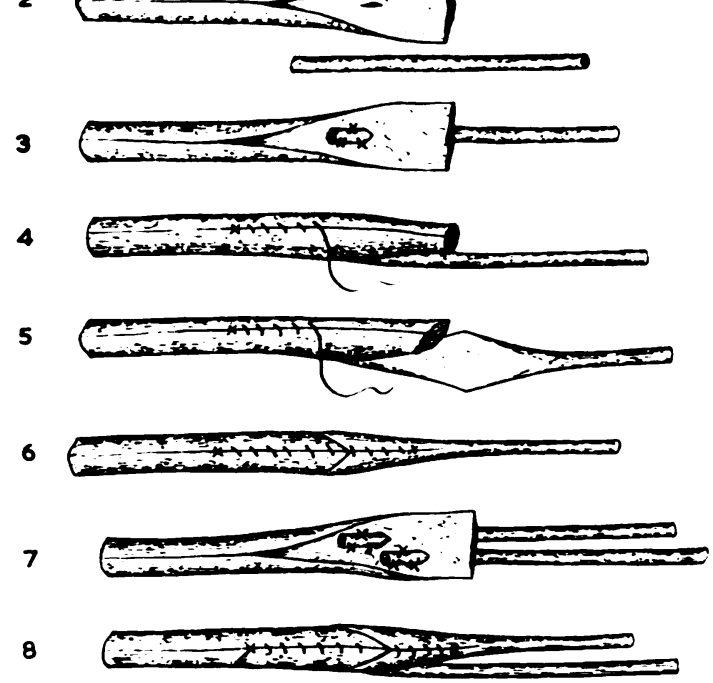

FIG. 2

Steps in the wrap-around method of tendon anastomosis. 1-6-Motor tendon and slender graft. 7-8-Motor tendon with two grafts. thickness of graft is lying in one part of the motor tendon. When it is important to have a uniform thickness and no bulge, a small part of the inside of the motor tendon is excised so that the total bulk of motor tendon and graft is the same as that of the original motor tendon.

The cut end of the motor tendon is then enclosed in the graft, which is opened out at the point at which it crosses the end of the motor tendon. If a thick graft has been used it may be slit open in the same way as the motor tendon. The best tendons for grafting are those of the plantaris and palmaris longus muscles, which are so thin that they possess no hilum but obtain their blood supply through the surface of the tendon; and they possess the peculiar property of a very large degree of lateral extensibility. Pilcher (1939) observed this property of the plantaris tendon when using it to repair hernias. The edges of the average plantaris can be pulled apart with needlepointed forceps, without the use of a knife,

into a sheet two inches wide. The end of the stoutest motor tendon can be completely engulfed by this beautiful glistening membrane, by suturing the edges together with 45 gauge stainless steel wire. When the anastomosis is complete no cut end of either graft or motor tendon should be visible in the wound. By cutting the motor tendon obliquely, the anastomosis tapers down gradually to the thickness of the graft.

We have recently developed a quick and simple method of removing a plantaris or palmaris tendon through one small incision. A stripper eighteen inches long terminates in an eye, a quarter of an inch in diameter, with a cutting edge (Fig. 3). The tendon is divided

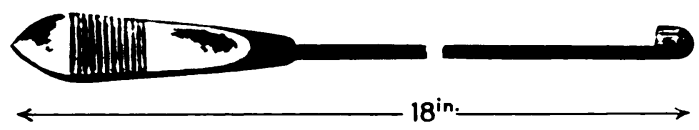

FIG. 3

Tendon stripper.

at its insertion and threaded into the eye of the stripper. The limb is held straight at the elbow or knee and the tendon kept stretched, while the stripper is thrust firmly up the limb until it cores out the tendon from inside the muscle, because all the muscle cannot pass through the eye. The tendon is then withdrawn through the incision. Bleeding is negligible and most patients from whom the plantaris has been taken are able to walk without pain within three or four days. 
The plantaris has an abundant paratenon which should be stripped back from the cut end in the form of a sleeve when an anastomosis is being done. This sleeve can then be rolled back to cover the suture area.

\section{OPERATION FOR CLAW HAND}

A good example of the value of tendon grafting is in intrinsic paralysis of the hand. In a previous communication (Brand 1958), in which we analysed the results in 564 fingers of the sublimis transfer operation of Stiles (1922) and Bunnell (1942), it was pointed out that the operation is a very powerful corrective for intrinsic paralysis of the fingers, but that the chief defect is the production of the opposite deformity-intrinsic overaction-in many patients. The reason for this is that the prime flexor of the proximal interphalangeal joint is removed and used as an extensor of the same joint. It seems that a finger with intrinsic paralysis cannot really spare its flexor sublimis, and that in the absence of this muscle as a flexor, it is too powerful to be used as an extensor.

We are now using a wrist-moving muscle extended by a many-tailed free graft for correction of intrinsic paralysis. At first toe extensor tendons were used as grafts and a flap was raised on the dorsum of the wrist for the anastomosis. Later the plantaris tendon and occasionally the palmaris were used and the need for the flap has been avoided by doing the operation entirely through short transverse incisions proximal and distal to the area of anastomosis. The grafts, after crossing the wrist, pass separately through the interosseous spaces along the route suggested by Fowler (1949), anterior to the deep transverse metacarpal ligament; they are sutured to the edge of the lateral band and dorsal expansion of each finger, proximal to the proximal interphalangeal joint. The tendon is inserted on the ulnar side of the index finger and on the radial side of the middle, ring and little fingers. The tendon to the little finger is usually passed through the third interosseous space and across the fourth metacarpal bone in the palm.

Recently the extensor carpi radialis longus has been used as the motor. It has been brought around the forearm and the grafts have been passed through the carpal tunnel.

Technique of operation-The extensor carpi radialis longus or brevis is divided at the distal end of the radius through a short transverse incision. It is withdrawn through a second transverse incision three and a half inches proximally, and laid on a wet towel. It is slit open along a natural plane of cleavage and the proximal ends of the tendon grafts are stitched to its inner surface. The tendon is then closed up around the ends of the grafts using a running stitch of 45 gauge stainless steel wire. One of the grafts is then stretched into a sheet and wrapped around the motor tendon, its edges held in position by the same filament of stainless steel wire. Tendon tunnelling forceps are then introduced at the first incision and passed subcutaneously to the proximal incision where the ends of the tendon grafts are grasped and pulled through so that the anastomosis lies under intact skin. Through dorsilateral incisions in the proximal segment of each finger the lumbrical tendon and lateral band are identified, and the tendon tunnelling forceps are now passed from this point through the palm to grasp a strand of tendon graft and withdraw it into the finger. Great care must be taken in this tunnelling process. The nose of the tunneller must first identify with certainty the deep transverse metacarpal ligament and pass it on the volar side for about half an inch. The handle of the tunnelling forceps is then lowered until it points dorsally in order to feel for a weak point in the interosseous membrane, through which the tunneller is thrust into the wound on the dorsum of the wrist. When all the tendon grafts are in position they are stitched at equal tension, suturing first the index finger and then the little finger and then the intermediate fingers. The tension chosen is such that the tendons are completely relaxed when the wrist is dorsiflexed 45 degrees, the metacarpo-phalangeal joints are flexed to 70 degrees, and the interphalangeal joints are straight (Fig. 4). This position is maintained for three weeks in a light plaster cast.

Re-education after operation-Although the late results of this operation are better than those of the sublimis transfer, the re-education is more difficult. It is most important not to allow 
the fingers to rest in the claw position during the period of re-education. We recommend splinting each finger with plaster or plastic gutter splints in the straight position, encouraging free metacarpo-phalangeal movement with resisted active extension of the wrist. The finger splints should be removed and reapplied daily until education is complete.

Defects in the operation-One defect of this operation is that dorsiflexion of the wrist slackens the graft. Therefore in re-education the patient has to prevent dorsiflexion while he contracts his dorsiflexor, which is difficult. The second defect is that adhesions are likely at the point where the graft perforates the interosseous membrane. As this point is distal to the wrist only a small range of tendon movement is necessary and therefore the result is satisfactory in spite of the adhesions. Both of these difficulties are overcome by the use of an alternative route we have been using recently.

Alternative route-The extensor carpi radialis longus tendon is used, detached at its insertion and withdrawn halfway up the forearm. It is then tunnelled deep to the brachioradialis to an incision on the front of the forearm three inches proximal to the wrist. The anastomosis to the grafts is now carried out. The tunneller is then introduced through a midpalmar incision and passed through the carpal tunnel into the forearm to bring the grafts into the palm, leaving the anastomosis proximal to the carpal tunnel. From this point each strand of graft is separately passed on to its finger destination (Fig. 5).

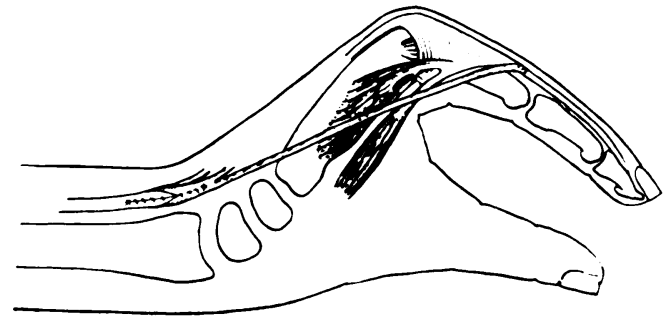

Fig. 4

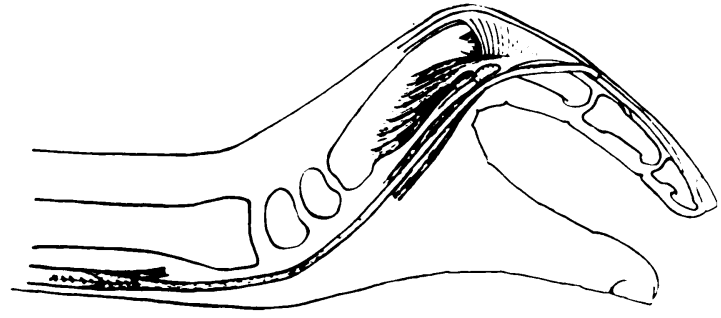

Fig. 5

Figure 4-Diagram showing the path of a tendon graft from the extensor carpi radialis brevis, through an interosseous space. Figure 5-Diagram showing the path of a tendon graft from the extensor carpi radialis longus, through the carpal tunnel. Re-education is easier when this route is used.

We have used this route (reported by Littler in his Case 6-b, 1949) in some of our recent operations, about ten of which are included here. We have found that although re-education after operation is somewhat easier than in the other method, there is sometimes a limitation of dorsiflexion of the wrist which weakens the grasp. There is also a theoretical danger that crowding the carpal tunnel may endanger the median nerve.

The flexor carpi radialis has also been used, extended by free grafts through the carpal tunnel, but the results have not been so good.

\section{MATERIAL}

Eight hundred and sixty-one clawed fingers in 246 hands have had tendon grafts using the extensor carpi radialis brevis as the motor muscle. All patients had leprosy, of whom fifty-three had a pure ulnar paralysis and 183 had a high ulnar and low median paralysis.

\section{METHOD OF ASSESSMENT}

We have used the same assessment as before (Brand 1958), but there has been a more critical interpretation of the terms " excellent" and "good." Measurements of the range of active, assisted, and passive movements of the fingers, and photographs in six standard positions, were taken before and after operation and again later during observation, so that each patient had eighteen standardised photographs (Fig. 6). The average length of observation 

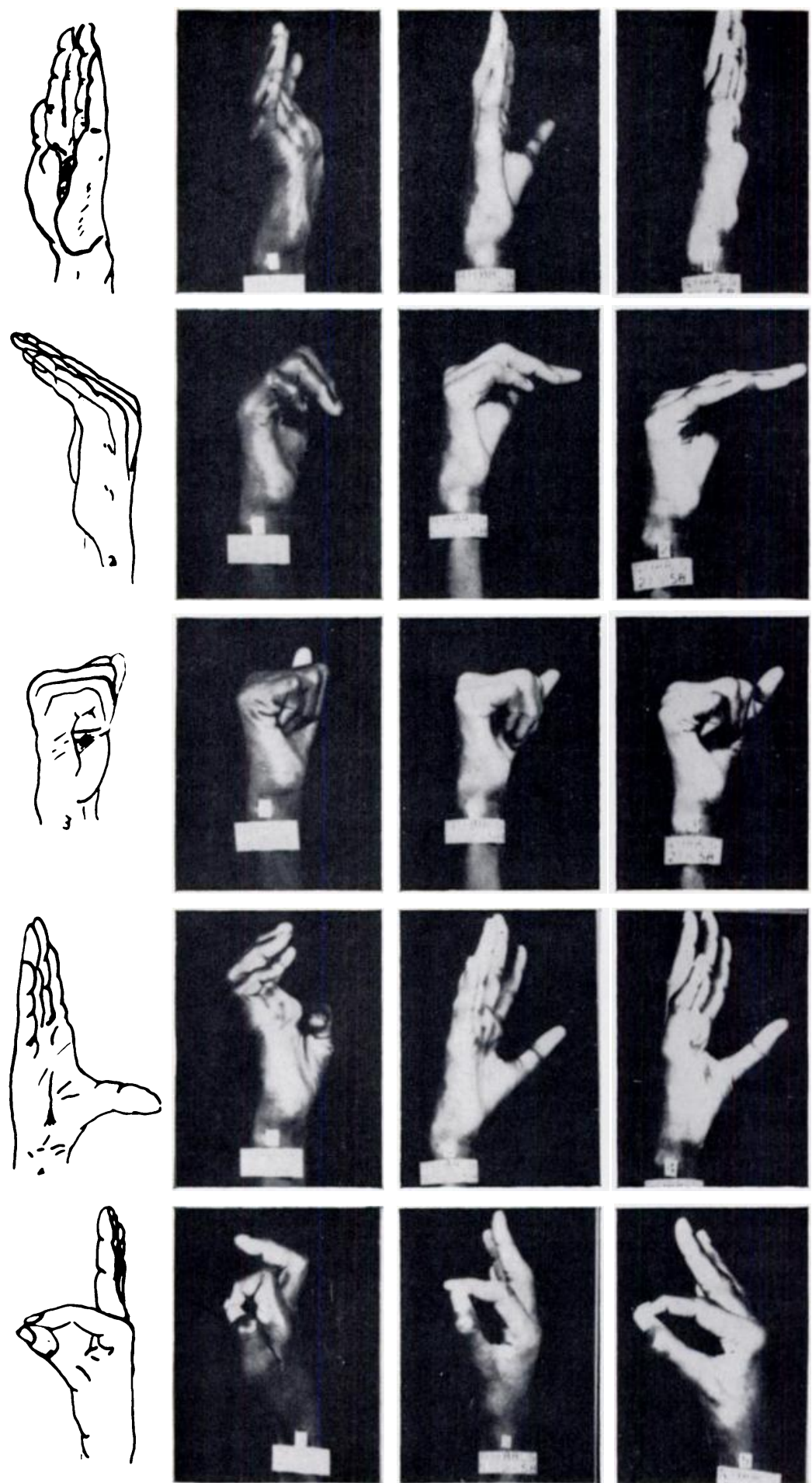

Fig. 6

A typical photographic record as used in every patient. The first column shows the diagram for imitation. The first photograph is the attempt before operation, the next that after operation, and last, the final result usually two or more years later. 
in this series is two years. It had been observed in the patients in whom the sublimis had been used that, although after operation the hand could achieve the lumbrical position and could open out fully there was frequently a difficulty in flexing the interphalangeal joints without previous flexion of the metacarpo-phalangeal joints; that is, it was impossible for the patient to put his hands into the old claw or "hook" position. This disability was not recorded in the sublimis assessment (Brand 1958) but a new assessment on the "hook"

TABLE I

Fully Open Hand Assessment in 861 Fingers

\begin{tabular}{|c|c|c|c|c|c|c|c|c|c|}
\hline \multicolumn{2}{|c|}{ Operations } & \multicolumn{2}{|c|}{ Excellent } & \multicolumn{2}{|c|}{ Good } & \multicolumn{2}{|c|}{ Fair } & \multicolumn{2}{|c|}{ Poor } \\
\hline Finger & Number & Number & Per cent & Number & Per cent & Number & Per cent & Number & Per cent \\
\hline Index & 185 & 90 & 49 & 60 & 32 & 33 & 18 & 2 & 1 \\
\hline Middle & 191 & 90 & 47 & 66 & 35 & 31 & 16 & 4 & 2 \\
\hline Ring & 243 & 118 & 48 & 87 & 36 & 36 & 15 & 2 & 1 \\
\hline Little & 242 & 126 & 52 & 78 & 32 & 36 & 15 & 2 & 1 \\
\hline
\end{tabular}

TABLE II

Lumbrical Position AsSessment in 861 Fingers

\begin{tabular}{|c|c|c|c|c|c|c|c|c|c|}
\hline \multicolumn{2}{|c|}{ Operations } & \multicolumn{2}{|c|}{ Excellent } & \multicolumn{2}{|c|}{ Good } & \multicolumn{2}{|c|}{ Fair } & \multicolumn{2}{|c|}{ Poor } \\
\hline Finger & Number & Number & Per cent & Number & Per cent & Number & Per cent & Number & Per cent \\
\hline Index & 185 & 72 & 39 & 74 & 40 & 37 & 20 & 2 & 1 \\
\hline Middle & 191 & 72 & 38 & 78 & 41 & 38 & 20 & 3 & 1 \\
\hline Ring & 243 & 98 & 40 & 107 & 44 & 35 & 15 & 3 & 1 \\
\hline Little & 242 & 120 & 50 & 89 & 37 & 30 & 12 & 3 & 1 \\
\hline
\end{tabular}

TABLE III

HoOK Position Assessment in 861 Fingers

\begin{tabular}{|c|c|c|c|c|c|c|c|c|c|}
\hline \multicolumn{2}{|c|}{ Operations } & \multicolumn{2}{|c|}{ Excellent } & \multicolumn{2}{|c|}{ Good } & \multicolumn{2}{|c|}{ Fair } & \multicolumn{2}{|c|}{ Poor } \\
\hline Finger & Number & Number & Per cent & Number & Per cent & Number & Per cent & Number & Per cent \\
\hline Index & 185 & 132 & 71 & 24 & 13 & 23 & 13 & 6 & 3 \\
\hline Middle & 191 & 138 & 72 & 18 & 9 & 26 & 14 & 9 & 5 \\
\hline Ring & 243 & 164 & 67 & 33 & 14 & 32 & 13 & 14 & 6 \\
\hline Little & 242 & 166 & 69 & 33 & 13 & 32 & 13 & 11 & 5 \\
\hline
\end{tabular}

position is included here. In Table V, "mechanics of closing" means the correct sequence of joint movement in making a fist.

After operation a hand to be called excellent must be able: 1) to open fully and freely at all joints with no degree of flexion limitation and with no hyperextension of the proximal interphalangeal joints and flexion of the terminal interphalangeal joints (intrinsic overaction Table I), 2) to flex at the metacarpo-phalangeal joint without flexion at the interphalangeal 
joints (lumbrical position Table II), 3) to flex at the interphalangeal joints without flexion at the metacarpo-phalangeal joints ("hook" position, Table III), 4) to close the fist fully with the finger tips tucked in (Table IV), 5) to open the hand without lateral deviation of the fingers or hyperextension of the metacarpo-phalangeal joints, 6) to achieve separate lumbrical movement of each finger, that is, each finger must be able to flex at the metacarpo-phalangeal joint without flexing the interphalangeal joints while the other fingers remain straight (Table V).

TABLE IV

Closed Fist Assessment in 246 Hands

\begin{tabular}{|c|c|c|c|c|c|c|c|}
\hline \multicolumn{2}{|c|}{ Excellent } & \multicolumn{2}{|c|}{ Good } & \multicolumn{2}{|c|}{ Fair } & \multicolumn{2}{|c|}{ Poor } \\
\hline Number & Per cent & Number & Per cent & Number & Per cent & Number & Per cent \\
\hline 145 & 59 & 42 & 17 & 51 & 21 & 8 & 3 \\
\hline
\end{tabular}

TABLE V

Mechanics of Closing in 246 Hands

\begin{tabular}{|c|c|c|c|c|c|c|c|}
\hline \multicolumn{2}{|c|}{ Excellent } & \multicolumn{2}{|c|}{ Good } & \multicolumn{2}{|c|}{ Fair } & \multicolumn{2}{|c|}{ Poor } \\
\hline Number & Per cent & Number & Per cent & Number & Per cent & Number & Per cent \\
\hline 217 & 88 & 20 & 8 & 9 & 4 & 0 & 0 \\
\hline
\end{tabular}

TABLE VI

Final AsSESSMENTS IN 246 Hands

\begin{tabular}{|c|c|c|c|c|c|c|c|c|}
\hline & \multicolumn{2}{|c|}{ Excellent } & \multicolumn{2}{|c|}{ Good } & \multicolumn{2}{|c|}{ Fair } & \multicolumn{2}{|c|}{ Poor } \\
\hline & Number & Per cent & Number & Per cent & Number & Per cent & Number & Per cent \\
\hline Appearance. & 62 & 26 & 112 & 45 & 68 & 27 & 4 & 2 \\
\hline Function & 107 & 43 & 81 & 33 & 54 & 22 & 4 & 2 \\
\hline Patient's statement & 64 & 26 & 119 & 48 & 59 & 24 & 4 & 2 \\
\hline Overall total. & 60 & 24 & 115 & 47 & 67 & 27 & 4 & 2 \\
\hline
\end{tabular}

It is perhaps surprising that individual lumbrical action is possible when a single muscle has been used for all four fingers. The fact that this is so reminds us that most of the movements of the fingers are powered by forearm muscles while the intrinsic muscles provide the balancing tone, which at finger level requires only a small amplitude. In a hand rated excellent, therefore, there is no abnormality in appearance or activity except that voluntary abduction and adduction of the fingers is not possible (Table VI, Fig. 7).

\section{RESULTS}

Even with the more critical standards of evaluation used in this study the final results as shown in Tables I to VI are substantially better than those of the sublimis transfer operation. Twenty-four per cent achieved an excellent result, 71 per cent achieved an excellent or good result and in only four hands was the result poor. These four, on further analysis, showed that the poor result was caused in one hand by a pre-operative defect in the extensor tendons and destruction of the dorsal expansion over the proximal interphalangeal joint, and in another by dislocation of the extensor tendon in the interphalangeal gutter. A third developed 


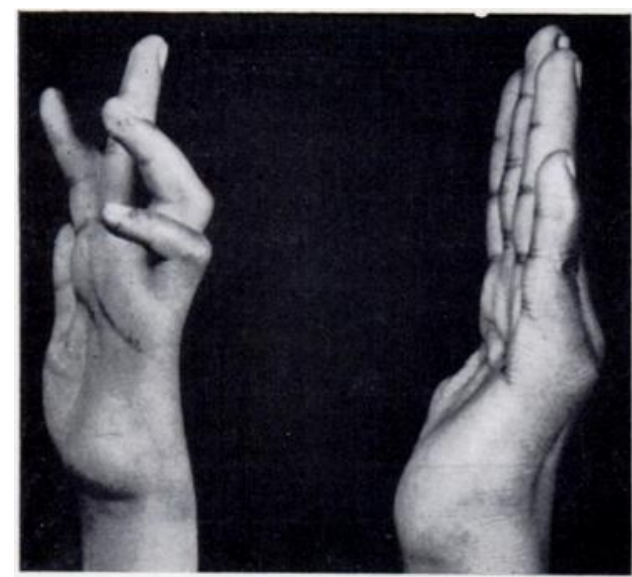

Fig. 7

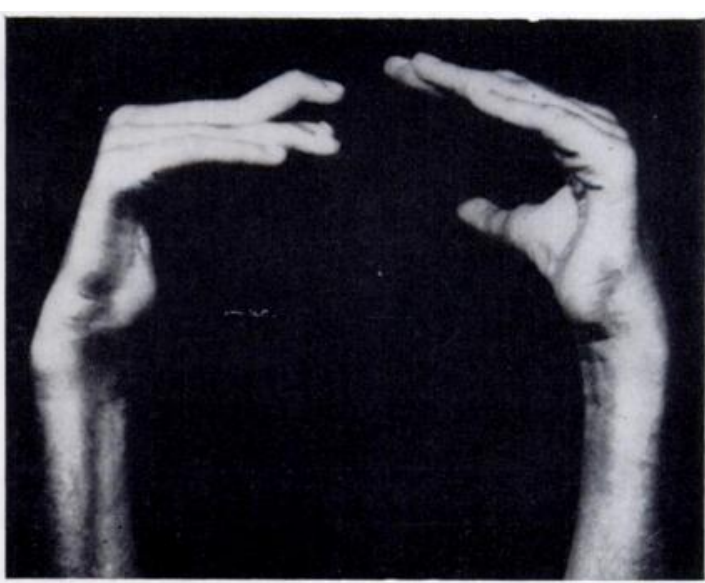

FIG. 8

Figure 7-A good result, three years after operation. Note slight bow-stringing of the graft on the back of the wrist on dorsiflexion.

Figure 8-Bilateral claw hands which had a sublimis transfer on the right hand in 1951 and a many-tailed graft on the left hand in 1957. Here the hands are held in the lumbrical position. The results are good on both sides but there is intrinsic overaction of the middle finger which had the sublimis transfer.

arthritis, and the limitation of movement was due to articular changes rather than failure of the tendon graft. The fourth hand developed infection at the site of anastomosis, causing adhesions and limitation of extension of all fingers.

It should be emphasised, however, that the results would not have been so good if it were not for the painstaking work of the physiotherapists. The results in a few patients who left the district without staying for re-education were inferior. In such patients it would have been better to have done a sublimis transfer (Fig. 8).

In this study there has been an element of case selection which was not present in the series on sublimis transfers. In a number of patients with contractures before operation which would not respond to physiotherapy and splintage, a sublimis transfer was chosen, because we believed that it would result in a stronger corrective force. In the past three years about 15 per cent of all claw hands have had sublimis transfers, and most of these have imperfect results because they had stiff fingers. Had these patients with contractions been operated upon by the free grafting technique the proportion of excellent and good results might have fallen from 71 per cent to 62 per cent.

Most of these operations have been done by different trainees in hand surgery, and in every patient the final assessments have been made by a member of the team who took no part in the operation.

\section{SUMMARY}

1. Tendon grafts, in order to survive, have to develop a blood supply from their immediate environment. This causes adhesions.

2. Their final range of movement is therefore a sum of the length to which these vascular adhesions will stretch, and the range of movement of the normal tissues to which the tendon has become adherent.

3. Thus it is important that the tendon graft should lie in a bed of yielding material, and that no unyielding structures should be divided in the same wound. When possible, blunt tunnelling between short transverse incisions is the method of choice for placement of grafts.

4. The most crippling adhesions are those that grow from an imperfectly sutured cut end of tendon, leaving it unsatisfied. A new method of tendon anastomosis designed to prevent such adhesions is described. 
5. A tendon grafting technique for intrinsic paralysis of the fingers in leprosy, using a radial extensor of the wrist as the motor and the plantaris tendon as the graft, is described.

6. A study of 861 fingers after the operation is presented.

7. The importance of re-education after operation is stressed.

I wish to thank my assistants and trainees for their meticulous care in record keeping which has made this study possible. I also wish to record my appreciation of the sterling work of our records clerk, Mr M. A. Furness. My thanks are due to CIBA for the production of a film demonstrating this grafting technique, now available in their film library. This study was assisted by a grant from the Indian Council of Medical Research. The tendon stripper and the tendon tunneller are made for us in India by the Indian Sterilizer Company, Madras 28, and in Britain by Messrs Chas. F. Thackray Ltd., Leeds 1.

\section{REFERENCES}

Brand, P. W. (1950): The Orthopaedic Care of Leprosy Patients. Journal of the Christian Medical Association, India, 25, 1.

BRand, P. W. (1952): The Reconstruction of the Hand in Leprosy. (Hunterian Lecture.) Annals of the Royal College of Surgeons of England, 11, 350.

Brand, P. W. (1954): Hand Reconstruction in Leprosy. In British Surgical Practice. Surgical Progress 1954, p. 117. London: Butterworth \& Co. (Publishers) Ltd.

Brand, P. W. (1954): The Place of Physical Medicine and Orthopaedic Surgery in Leprosy. Leprosy Review, $25,5$.

Brand, P. W. (1955): The Value of Surgical and Physiotherapeutic Measures in Leprosy. Leprosy in India, $27,131$.

BRand, P. W. (1956): Treatment of Leprosy. II. The Role of Surgery. New England Journal of Medicine, 254, 64.

Brand, P. W. (1958): Paralytic Claw Hand. Journal of Bone and Joint Surgery, 40-B, 618.

Brand, P. W. (1959): Deformity in Leprosy. Orthopaedic Principles and Practical Methods of Relief. In Leprosy in Theory and Practice, p. 265. Edited by R. G. Cochrane. Bristol: John Wright \& Sons Ltd.

BunNell, S. (1932): Contractures of the Hand from Infections and Injuries. Journal of Bone and Joint Surgery, $14,27$.

Bunnell, S. (1942): Surgery of the Intrinsic Muscles of the Hand Other Than Those Producing Opposition of the Thumb. Journal of Bone and Joint Surgery, 24, 1.

Bunnell, S. (1956): Surgery of the Hand. Third edition. Philadelphia: J. B. Lippincott Company.

Denio, K. (1897): Ueber die Lepra anaesthetica und den pathogenetischen Zusammenhang ihrer Krankheitserscheinungen. Mittheilungen und Verhandlungen der internationalen wissenschaftlichen Lepra-Conferenz zu Berlin im October 1897. Band 2, p. 85. Berlin: August Hirschwald.

Eyler, D. L., and Markee, J. E. (1954): The Anatomy and Function of the Intrinsic Musculature of the Fingers. Journal of Bone and Joint Surgery, 36-A, 1.

Fowler, S. B. (1949): Extensor Apparatus of the Digits. Journal of Bone and Joint Surgery, 31-B, 477.

Glissan, D. J. (1932): The Use of the Plantaris Tendon in Certain Types of Plastic Surgery. Australian and New Zealand Journal of Surgery, 2, 64.

Goldner, J. L. (1953): Deformities of the Hand Incidental to Pathological Changes of the Extensor and Intrinsic Muscle Mechanisms. Journal of Bone and Joint Surgery, 35-A, 115.

Harris, C., Jun., and Riordan, D. C. (1954): Intrinsic Contracture in the Hand and its Surgical Treatment. Journal of Bone and Joint Surgery, 36-A, 10.

Kaplan, E. B. (1953): Functional and Surgical Anatomy of the Hand. Philadelphia: J. B. Lippincott Company.

Littler, J. W. (1949): Tendon Transfers and Arthrodeses in Combined Median and Ulnar Nerve Paralysis. Journal of Bone and Joint Surgery, 31-A, 225.

Mason, M. L., and Shearon, C. G. (1932): The Process of Tendon Repair; an Experimental Study of Tendon Suture and Tendon Graft. Archives of Surgery, 25, 615.

Mayer, L. (1916): The Physiological Method of Tendon Transplantation. Surgery, Gynecology and Obstetrics, 22, 182.

Ney, K. W. (1921): A Tendon Transplant for Intrinsic Hand Muscle Paralysis. Surgery, Gynecology and Obstetrics, 33, 342.

Pilcher, R. (1939): Repair of Hernia with Plantaris Tendon Graft. Archives of Surgery, 38, 16.

Pulvertaft, R. G. (1956): Tendon Grafts for Flexor Tendon Injuries in the Fingers and Thumb. A Study of Technique and Results. Journal of Bone and Joint Surgery, 38-B, 175.

Riordan, D. C. (1954): Tendon Transplantations in Median-Nerve and Ulnar-Nerve Paralysis. Journal of Bone and Joint Surgery, 35-A, 312.

Stiles, Sir H. J., and Forrester-Brown, M. F. (1922): Treatment of Injuries of the Peripheral Spinal Nerves, p. 180. London: Henry Frowde and Hodder \& Stoughton.

VOL. 43 B, NO. 3, AUGUST 1961 